\title{
Perdita Considers Dessert
}

The mountain sits in levels like a wedding cake for a bride that never showed.

Really, the cake was in bad taste anyway, weighted with countless figures in a drooping frosting:

the sugary nude, bowed forever beneath boulders

like camels, with the buttock-crack beneath that rising hump;

the envious, under brains twisted like walnuts their eyes sewn closed;

the angry, with open eyes in a stinging smoke.

What would the style of this cake be called?

Baroque? Hudson kitsch?

Much more attractive than a display of sinners would have been to let everyone go to heaven, tucking in their feathers

with the fastidiousness of a swan. 